\title{
POPULATION-BASED APPROACH TO ANALYZE SPARSE SAMPLING DATA IN BIOPHARMACEUTIC AND PHARMACOKINETIC STUDIES USING NONMEM AND MONOLIX
}

\begin{abstract}
Akhmad Kharis Nugroho ${ }^{1} *$, Arief Rahman Hakim², Lukman Hakim²
1Department of

Pharmaceutics, Faculty of Pharmacy, Universitas Gadjah Mada, Yogyakarta 55281, Indonesia 2 Department of Pharmacology and Clinical Pharmacy, Faculty of Pharmacy, Universitas Gadjah Mada, Yogyakarta 55281, indonesia

Submitted: $14-07-2017$

Revised: $11-11-2017$

Accepted: $12-12-2017$

*Corresponding author Akhmad Kharis Nugroho

Email:

a.k.nugroho@ugm.ac.id

ABSTRACT

Although it has been developed since 1972, the application of a population-based modeling approach in Indonesia, particularly to analyze biopharmaceutic and pharmacokinetic data remains very limited. This study was aimed to evaluate the performance of Monolix and NONMEM, two of the popular software packages in a population-based modeling approach, to analyze the limited data (sparse data) of the time profiles of the simulated plasma drug concentration of a theoretical compound. Monolix and NONMEM were used to model the limited data (40 data points) resulting from random selection of 180 data points of plasma drug concentrations (Cp) in 20 subjects at $0.25 ; 0.5$; $0.75 ; 1 ; 1.5 ; 3 ; 6 ; 12$ and 18 hours after per-oral administration of a $100 \mathrm{mg}$ of a theoretical compound. Values of the absorption rate constant $(\mathrm{Ka})$, the elimination rate constant (Kel) and the distribution volume $(\mathrm{Vd})$ of sparse data estimated using Monolix and NONMEM, were compared to the respective values of rich data obtained by a conventional two-stage approach using PKSolver. The calculation system of a nonlinear mixed effect model in Monolix and NONMEM, successfully describes the sparse data, based on the visual evaluation of the goodness of fit. Comparison of parameter estimates of population values in Monolix and NONMEM are in the range of 94 to $108 \%$ of the real values of the rich data analyzed by PKSolver. A population-based modeling can adequately describe limited or sparse data, demonstrating its capability as an important tool in clinical studies, involving patients.
\end{abstract}

Key words: population-based approach, nonlinear mixed effect model, sparse data, Monolix, NONMEM

\section{INTRODUCTION}

Population-based modeling approach has been developed since 1972, by introduction and development of the so called nonlinear mixed effect model (Sheiner et al., 1972). Briefly, in this method, a certain parameter or variable, for example the rate constant of absorption, $\mathrm{Ka}$, is considered determined by a population or fixed effect value and an interindividual variability, resulted in a different $\mathrm{Ka}$ parameter value in each subject. As such approach directly focuses on the population data, it allows analyses based on the sparse sampling data, which is commonly obtained in clinical studies involving patients (Sheiner et al., 1972).
This is beneficial, in contrast to a conventional two stage approach which requires a richer data and cannot analyze such limited data. The nonlinear mixed effect model is also able to correlate a certain parameter such as $\mathrm{Ka}$, clearance $(\mathrm{CL})$, distribution volume $(\mathrm{Vd})$ or elimination rate constant (Kel) to certain covariates such as sex, age, body weight in a quantitative manner, allowing a better description and correlation of the population data to those covariates (Jonsson and Karlsson, 1998; Wählby et al., 2001).

NONMEM was introduced by Lewis Sheiner, Stuart Beal and NONMEM Project Group at University of California as the first software package capable to handle such 
complex calculation in this nonlinear mixed effect modeling approach (Dartois et al., 2007; Sheiner et al., 1972). The implementation and application of NONMEM in this approach has opened a new era in population-based pharmacokinetic studies, allowing quantitative analyses of the clinical data such as obtained in a therapeutic drug monitoring (TDM) (Parke and Charles, 1998; Sandström et al., 2001; Shaker et al., 2013). In addition to a fast computation, NONMEM also provides robust, accurate and precise maximum likelihood calculation (Plan et al., 2012). To date, NONMEM is still considered to be the gold standard software in population pharmacokinetic-pharmacodynamic modeling (Keizer et al., 2013). Currently, NONMEM is commercially managed and further developed by ICON plc. (Frame, 2006).

In addition, there are several software alternatives for population-based approach which are free or free for academic use. One of them is Monolix, developed since 2003. It is based on SAEM (Stochastic Approximation Effect Model) algorithm using MATLAB library engine as its core (Sun and Li, 2011). In certain pharmacokinetic-pharmacodynamic modeling case, particularly with a complex differential equations structure, SAEM algorithm in Monolix could be considered more powerful than FOCE with interaction in NONMEM (Chan et al., 2011). Monolix is developed based on a thorough statistical theory and could provide a fast and efficient calculation in sparse data conditions with a large inter individual variability (Lavielle and Mentré, 2007). Monolix, which is currently maintained and developed by Lixoft company, has graphical user interface allowing an easier and a more practical use of the software in population modeling (Lavielle, 2014).

Despite of all advantages provided by the population based approach, its implementation is however still very limited in Indonesia. More studies implementing the population-based approach should be carried out and reported to introduce and familiarize more researchers with this approach and applications. This research was focused on studying the power of Monolix and NONMEM to analyze the sparse sampling data, which were taken up randomly from a rich data of the simulated drug plasma concentration $(\mathrm{Cp})$ versus time profile of a hypothetical compound.

\section{MATERIAL AND METHODS \\ Preparation of data simulation}

We simulated the data of $\mathrm{Cp}$ versus time after per-oral administration of a $100 \mathrm{mg}$ of a hypothetical compound ( $\mathrm{f}=1$ ) using Microsoft Excel 2013 (running on Windows 10 machine) (Liengme, 2015). This step resulted in creation of a rich Cp data profiles, i.e. 180 data point of 20 subjects at 9 time points (at $0.25 ; 0.5 ; 0.75 ; 1$; $1.5 ; 3 ; 6 ; 12$ and $18 \mathrm{~h}$ ) for each subject with the parameters of $\mathrm{Ka}$, Kel and $\mathrm{Vd}$ at values of $1.87 \pm 0.15$ per hour, $0.26 \pm 0.01$ per hour and $333 \pm 38 \mathrm{~L}$, respectively. These rich data are considered the reference or real data (Figure 1 panel A).

Furthermore, the sparse sampling data were originated from the random selection of those rich data by choosing 1 to 3 data per subject. This resulted in 40 data points from 20 subjects (Figure 1 panel B).

\section{Data analysis}

Monolix (stand alone, version 4.3.2, running on Windows 10 machine) (Chan et al., 2011) and NONMEM (version 7.3, using Gfortran compiler, directed using PLTTools lite version 5.4.1) (Frame, 2006) were used to model the limited sparse sampling data.

Inter-individual variability was modelled by an exponential error model as written in equation 1 .

$P i=\theta . \exp (\eta i) \ldots \ldots \ldots \ldots \ldots \ldots \ldots(1)$ in which $\theta$ is the population value or the fixed effect parameter P. Pi is the individual estimate value and $\eta_{\mathrm{i}}$ is the inter-individual variation, for which the values are assumed to be independently and normally distributed with mean zero and variance $\omega^{2}$. The interindividual variability was applied for $\mathrm{Ka}$, $\mathrm{Vd}$ and Kel.

The residual error was characterised by the additive error model in equation 2 .

$\boldsymbol{F p}_{i j}=\boldsymbol{F o}_{i j}+\varepsilon_{1} \ldots \ldots \ldots \ldots \ldots \ldots(2)$ where $\mathrm{Fp}_{\mathrm{ij}}$ is the prediction of the $\mathrm{j}^{\text {th }}$ evaluated functions (Cp), $\mathrm{Fo}_{\mathrm{ij}}$ is the measured value of the evaluated function (Cp), and $\varepsilon$ represents 

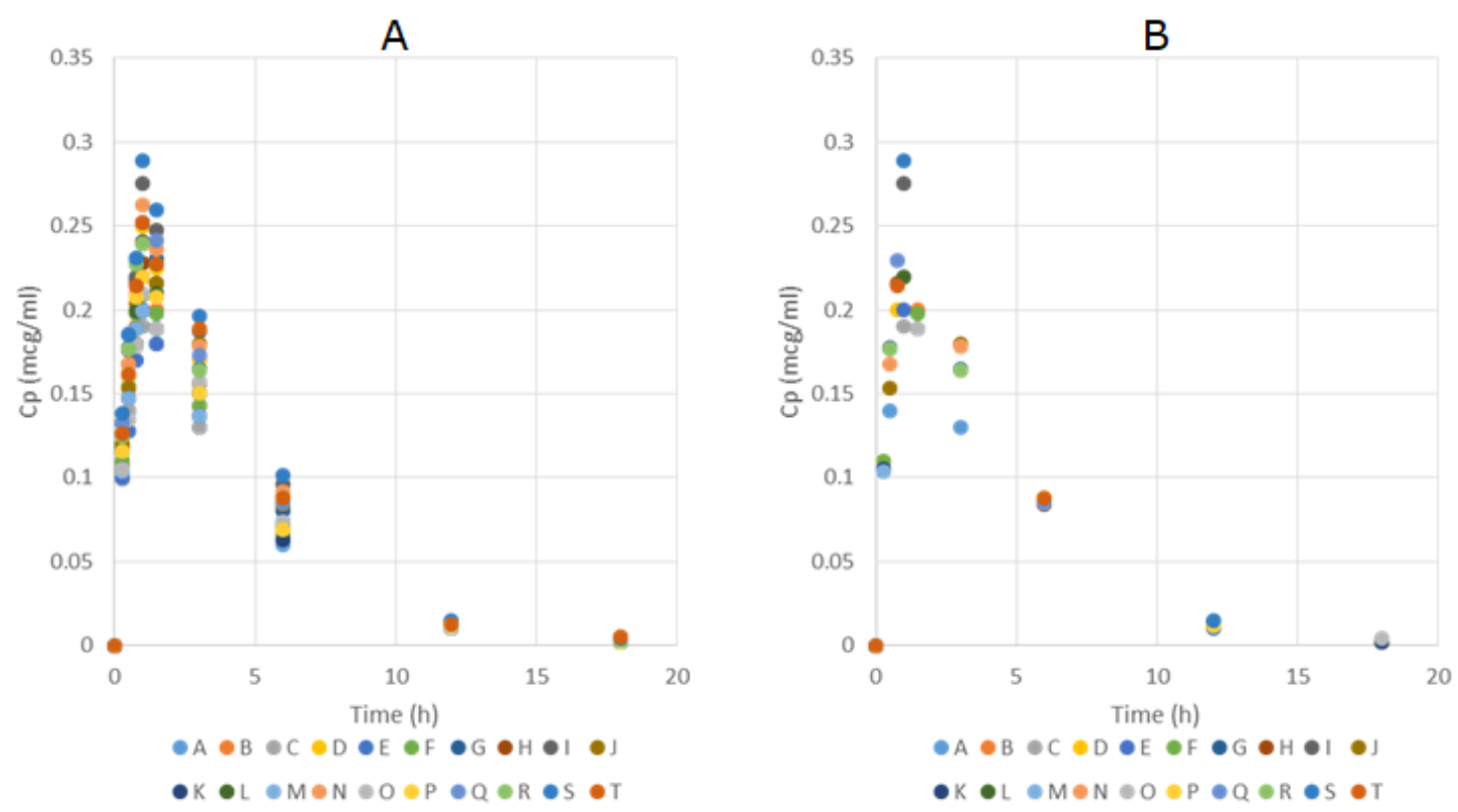

Figure 1. Data of the rich/real data (panel A) and the sparse data (panel B) of the simulated Cp versus time profile of a $100 \mathrm{mg}$ theoretical compound delivered by per-oral route $(\mathrm{f}=1)$.

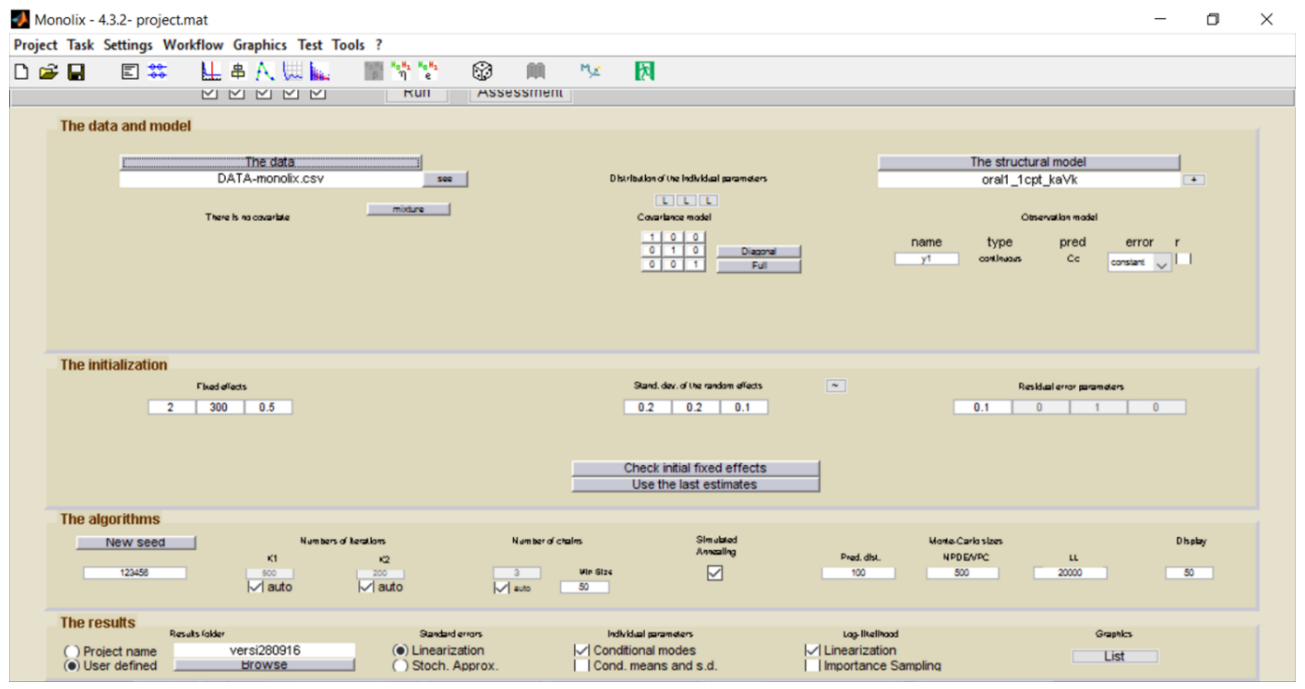

Figure 2. Configuration of Monolix analyses of the sparse data

the residual deviation of the predicted from the observed value and is assumed to be independently and normally distributed with mean zero and variance $\sigma^{2}$. The analysis of the population parameters $\theta, \omega^{2}$, and $\sigma^{2}$ was performed using SAEM algorithm and the first-order with interaction method (FOCE) in Monolix and NONMEM respectively.
In Monolix, analyses were performed using the structural one compartment oral model with $\mathrm{Ka}$, Kel and $\mathrm{Vd}$ parameters provided in Monolix library. No covariate was applied while the covariance implemented the default diagonal pattern. Data were assumed to follow a $\log$ normal distribution. Calculation was performed using 2 (per hour), 300 (L) and 0.5 (per hour) as initial estimates of $\mathrm{Ka}, \mathrm{Vd}$ and 


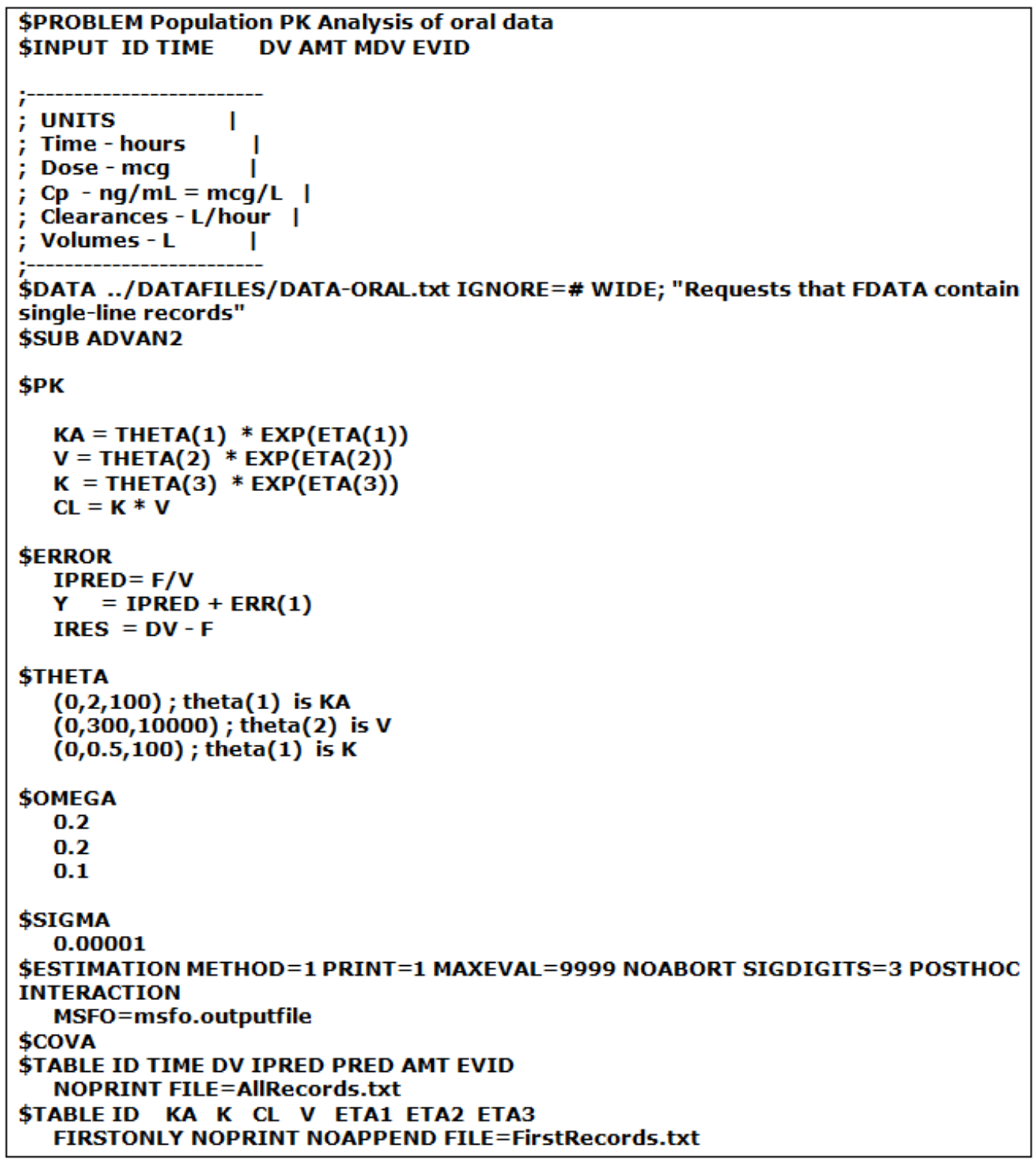

Figure 3. Listing code of NONMEM analyses of the sparse data

Kel respectively. The initial estimates of the random error (eta) were $0.2,0.2$ and 0.1 for $\mathrm{Ka}$, $\mathrm{Vd}$ and Kel respectively. Additive error model was calculated with the initial values of sigma of 0.1 (Figure 2).

In NONMEM, analyses were performed using the provided one compartment oral model (ADVAN 2) with $\mathrm{Ka}$, Kel and $\mathrm{Vd}$ parameters provided in NONMEM PK library. No covariate was applied while the covariance implemented the default diagonal pattern. Data were assumed to follow a log normal distribution. Calculation was performed using 2 (per hour), 300 (L) and 0.5 (per hour) as initial estimates of $\mathrm{Ka}, \mathrm{Vd}$ and $\mathrm{Kel}$ respectively. The initial estimates of the random error (eta) were $0.2,0.2$ and 0.1 for $\mathrm{Ka}, \mathrm{Vd}$ and $\mathrm{Kel}$ respectively. Additional residual error model was used with the initial values of 0.00001 (Figure 3) .

The individual parameter values of $\mathrm{Ka}$, Kel and Vd obtained from the analyses of the sparse sampling data using Monolix and NONMEM were compared to the individual prediction of $\mathrm{Ka}$, Kel and $\mathrm{Vd}$ obtained by the conventional (two stage approach) analyses of 


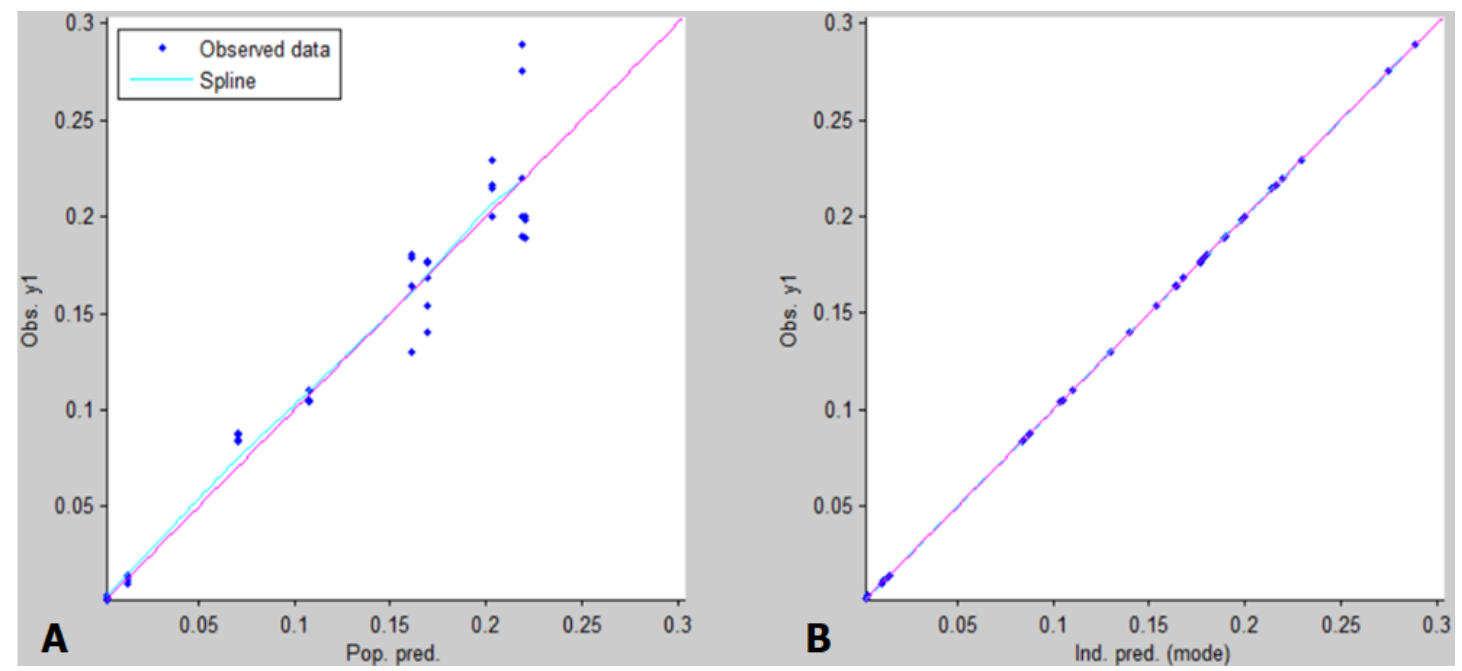

Figure 4. Goodness of fit analyses of the sparse data using Monolix based on correlation of population prediction of $\mathrm{Cp}$ (panel A) and post-hoc individual prediction (panel B) versus the values of $\mathrm{Cp}$
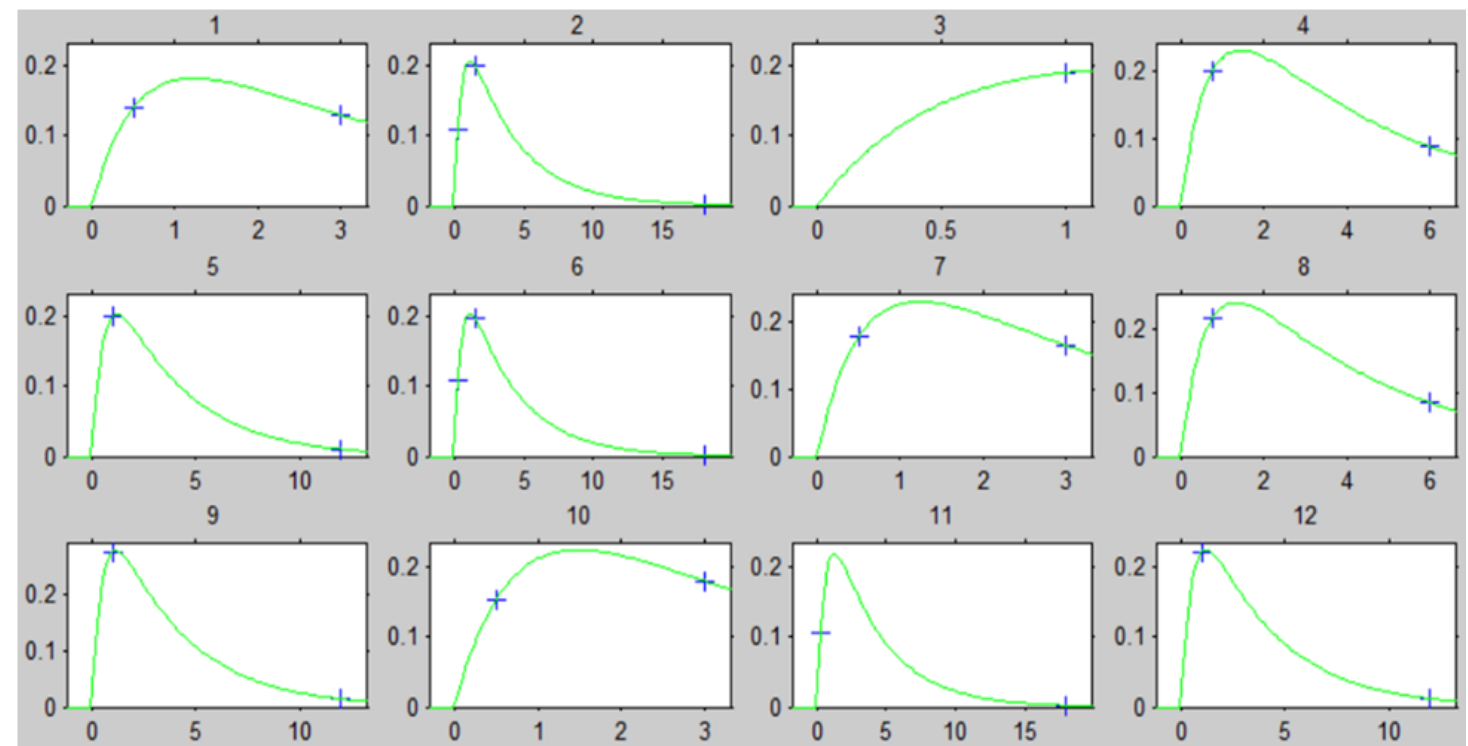

Figure 5. Typical example of the pos-hoc individual fitting of the sparse data using Monolix

the rich data using PKSolver version 2.0 (Zhang et al., 2010). Due to non-normal distribution of some data (tested based on Shapiro-Wilkes method using OpenStat 2012), the comparison of means of $\mathrm{Ka}$, Kel and $\mathrm{Vd}$ of the sparse sampling data to the rich data was performed based on the Wilcoxon matchedpairs method using OpenStat version 2012 (Miller, 2012).

\section{RESULTS AND DISCUSSION}

Monolix analyses the sparse data in several type of calculation. One of the important part of the goodness of fit is based on presentation of population prediction versus data as well as individual prediction versus data (Mohammed et al., 2012; Owen and FiedlerKelly, 2014; Zheng et al., 2014) (Figure 4). Although the population prediction of $\mathrm{Cp}$ data 
A

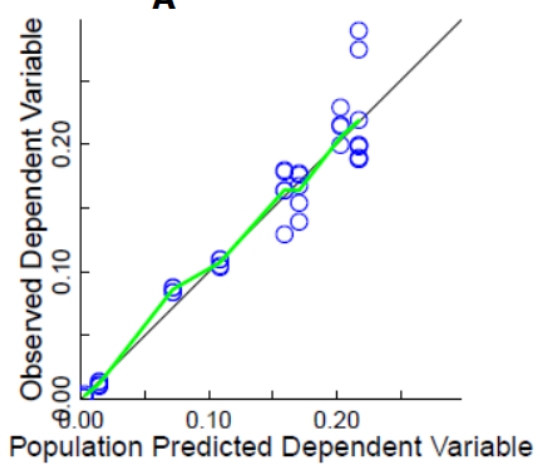

B

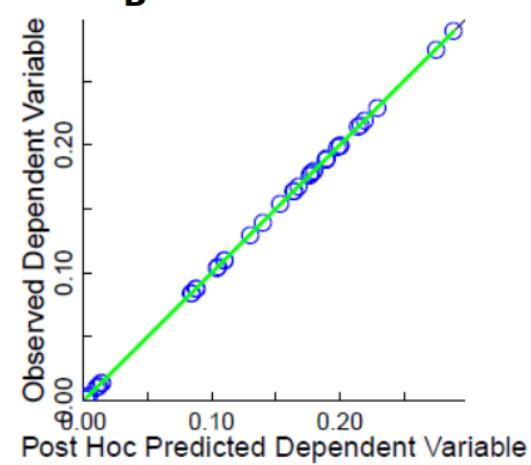

Figure 6. Goodness of fit analyses of the sparse data using NONMEM based on correlation of population prediction of $\mathrm{CP}$ (panel A) and post-hoc individual prediction (panel B) versus the values of $\mathrm{Cp}$
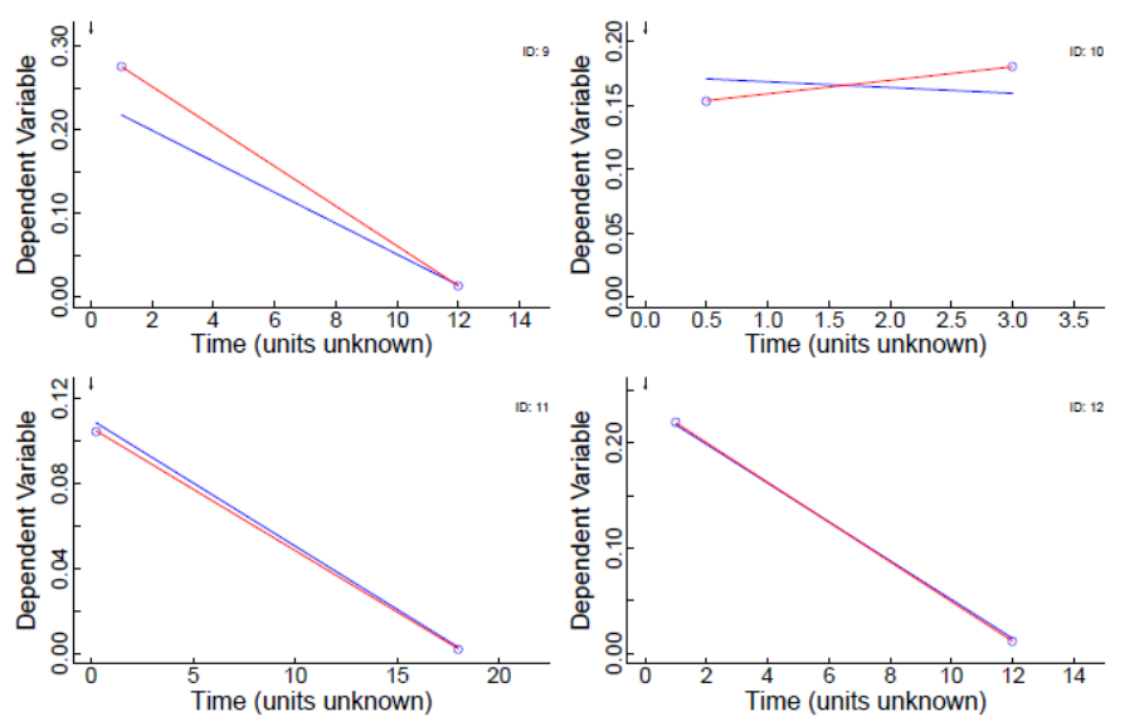

Figure 7. Typical example of the post-hoc individual fitting (red line) and the population prediction fitting (blue line) of the sparse data using NONMEM

does not clearly demonstrate a random distribution around the line of identity, there is no certain pattern or shape (for example sigmoid, or shoe shape) indicating a good population fitting. Furthermore, the individual prediction is presented mostly on the line of identity. This indicates that systematically the model could adequately describe all of the limited, sparse sampling data. Good individual fitting is also occured from the individual data fit (Figure 5), which represents the good systematic description of the model to the data (Owen and Fiedler-Kelly, 2014).
Similarly, NONMEM models the sparse data very adequately. Figure 6 demonstrates the good fitting of both population as well as the individual post hoc estimation of the observed data. Good individual fitting (Figure 7), which demonstrates the good description of the model to the sparse data.

Furthermore, we analyze the individual pharmacokinetic parameter values of $\mathrm{Ka}, \mathrm{Vd}$ and Kel of the sparse data to the rich data using the Wilcoxon matched-pairs method by comparing each parameter values of the same subject (Table I). Although in all comparisons, 
Table I. Comparison of parameter estimation of $\mathrm{Ka}, \mathrm{Vd}$ and $\mathrm{Kel}$ of the sparse data by Monolix and NONMEM to the estimation values of the real/rich data. Data are presented as mean \pm standard deviation of 20 subjects.

\begin{tabular}{cccccccc}
\hline \multirow{2}{*}{ Parameter } & $\begin{array}{c}\text { Rich data - } \\
\text { PK Solver }\end{array}$ & \multicolumn{2}{c}{ Sparse data - Monolix } & \multicolumn{3}{c}{ Sparse data - NONMEM } \\
\cline { 2 - 8 } & $\begin{array}{c}\text { Estimated } \\
\text { value }\end{array}$ & $\begin{array}{c}\text { Estimated } \\
\text { value }\end{array}$ & \% rasio\# & p* & $\begin{array}{c}\text { Estimated } \\
\text { value }\end{array}$ & \% rasio\#\# & \multirow{2}{*}{$\mathbf{p}^{*}$} \\
\hline Ka & $1.87 \pm 0.15$ & $1.76 \pm 0.19$ & $94.2 \pm 9.8$ & $\mathrm{p}<0.05$ & $1.83 \pm 0.18$ & $98.1+10.2$ & $\mathrm{p}>0.05$ \\
Vd & $333 \pm 39$ & $319 \pm 38$ & $95.8 \pm 4.8$ & $\mathrm{p}<0.05$ & $327 \pm 38$ & $98.2 \pm 4.7$ & $\mathrm{p}>0.05$ \\
Kel & $0.26 \pm 0.01$ & $0.28 \pm 0.01$ & $107.8 \pm 5.7$ & $\mathrm{p}<0.05$ & $0.27 \pm 0.02$ & $104.3 \pm 6.4$ & $\mathrm{p}<0.05$ \\
\hline
\end{tabular}

*based on Wilcoxon matched-pairs test; $\# \%$ ratio of $\mathrm{Ka}, \mathrm{Vd}$ and Kel parameter values in sparse data estimated using Monolix to the respective parameters values in rich data by PK-Solver; \#\# \% ratio of $\mathrm{Ka}, \mathrm{Vd}$ and Kel parameter values in sparse data estimated using NONMEM to the respective parameters values in rich data by PK-Solver

the differences are significant $(\mathrm{p}<0.05)$, the mean values of the sparse sampling data of those 20 subjects for $\mathrm{Ka}, \mathrm{Vd}$ and $\mathrm{Kel}$ estimated by Monolix are in the ratio of $94 \%, 96 \%$ and $108 \%$ over the real/rich data.

Furthermore, NONMEM better estimates the values of those parameters. The mean values of the sparse sampling data of those 20 subjects for Ka, Vd and Kel (Table I), are in the ratio of 98,98 and $104 \%$ over the real/rich data. The difference is significant $(\mathrm{p}<0.05)$ only in Kel comparison. Thus, both Monolix and NONMEM can predict the parameter values of $\mathrm{Ka}, \mathrm{Vd}$ and $\mathrm{Kel}$ in the range of less than $10 \%$. These facts indicate a good capability of both Monolix and NONMEM to adequately describe the sparse data.

Such capability is very important to complement a therapeutic drug monitoring of certain drugs. In such cases, a sparse data is collected from patients (Parke and Charles, 1998; Shaker et al., 2013). This present study indicates that Monolix and NONMEM can adequately describe the simulated sparse data, limited only to one up to three samples per subject. Further studies are required in each drug of interest to study the best scenario in the therapeutic drug monitoring performed.

With this concern, it is important to obtain sample at different time point in each patients. In compilation, such strategy results in a complete figure of the absorption, distribution and elimination phases of all patients population. This allows Monolix,
NONMEM or other software packages to accurately estimate pharmacokinetic parameters in each subject. Those parameters are among the important aspects to evaluate whether the outcome of therapy has been reached or a new dose regimen is required.

\section{CONCLUSION}

Population-based modeling approach can adequately describe limited, sparse sampling data to estimate the model parameters. Estimation of those parameters could facilitate a better therapeutic outcome for patients by means of therapeutic drug monitoring resulting in adequate justification for dose regimen during therapy. Monolix as a free (for academic) software package has a comparable performance to NONMEM, which opens broader application for optimizing therapeutic outcome in Indonesia.

\section{ACKNOWLEDGMENT}

This research was supported by Hibah Penelitian Unggulan Perguruan Tinggi, a research grant scheme provided by the Ministry of Research, Technology and Higher Education, the Republic of Indonesia, year 2016.

\section{REFERENCES}

Chan, PLS., Jacqmin, P., Lavielle, M., McFadyen, L., Weatherley, B., 2011. The use of the SAEM algorithm in MONOLIX software for estimation of population pharmacokinetic- 
pharmacodynamic-viral dynamics parameters of maraviroc in asymptomatic HIV subjects. J. Pharmacokinet. Pharmacodyn. 38:41-61.

Dartois, C., Lemenuel-Diot, A., Laveille, C., Tranchand, B., Tod, M., Girard, P., 2007. Evaluation of uncertainty parameters estimated by different population PK software and methods. J. Pharmacokinet. Pharmacodyn. 34:289-311.

Frame, B., 2006. Mixture Modeling with NONMEM V, in: Ette, E.I., Williams, P.J. (Eds.), Pharmacometrics: The Science of Quantitative Pharmacology. John Wiley \& Sons, Inc, Hoboken, New Jersey, pp. 723-757.

Jonsson, EN., Karlsson, M.O., 1998. Automated covariate model building within NONMEM. Pharm. Res. 15:14631468.

Keizer, RJ., Karlsson, MO., Hooker, A., 2013. Modeling and Simulation Workbench for NONMEM: Tutorial on Pirana, PsN, and Xpose. CPT Pharmacomet. Syst. Pharmacol. 2:e50.

Lavielle, M., 2014. Mixed Effects Models for the Population Approach: Models, Tasks, Methods and Tools. CRC Press.

Lavielle, M., Mentré, F., 2007. Estimation of Population Pharmacokinetic Parameters of Saquinavir in HIV Patients with the MONOLIX Software. J. Pharmacokinet. Pharmacodyn. 34:229-249.

Liengme, B., 2015. A Guide to Microsoft Excel 2013 for Scientists and Engineers. Academic Press.

Miller, W., 2012. OpenStat Reference Manual. Springer Science \& Business Media.

Mohammed, BS., Engelhardt, T., Cameron, G.A., Cameron, L., Hawksworth, G.M., et al., 2012. Population pharmacokinetics of single-dose intravenous paracetamol in children. Br. J. Anaesth. 108:823-829.

Owen, JS., Fiedler-Kelly, J., 2014. Introduction to population pharmacokinetic/ pharmacodynamic analysis with nonlinear mixed effects models. Wiley, Hoboken, New Jersey.
Parke, J., Charles, BG., 1998. NONMEM Population Pharmacokinetic Modeling of Orally Administered Cyclosporine From Routine Drug Monitoring Data After Heart Transplantation: Ther. Drug Monit. 20:284-293.

Plan, EL., Maloney, A., Mentré, F., Karlsson, MO., Bertrand, J., 2012. Performance comparison of various maximum likelihood nonlinear mixed-effects estimation methods for dose-response models. AAPS J. 14:420-432.

Sandström, M., Karlsson, M.O., Ljungman, P., Hassan, Z., Jonsson, E.N., et al., 2001. Population pharmacokinetic analysis resulting in a tool for dose individualization of busulphan in bone marrow transplantation recipients. Bone Marrow Transplant. 28:657-664.

Shaker, E., Hamadi, S., Idkaidek, N., E Blakey, G., Al-Saleh, A., 2013. Therapeutic Drug Monitoring and Population Pharmacokinetics of Digoxin in Jordanian Patients. Am. J. Pharmacol. Sci. 1:15-21.

Sheiner, LB., Rosenberg, B., Melmon, KL., 1972. Modelling of individual pharmacokinetics for computer-aided drug dosage. Comput. Biomed. Res. 5:411459.

Sun, X., Li, J., 2011. PKreport: report generation for checking population pharmacokinetic model assumptions. BMC Med. Inform. Decis. Mak. 11:31.

Wählby, U., Jonsson, E.N., Karlsson, M.O., 2001. Assessment of actual significance levels for covariate effects in NONMEM. J. Pharmacokinet. Pharmacodyn. 28:231-252.

Zhang, Y., Huo, M., Zhou, J., Xie, S., 2010. PKSolver: An add-in program for pharmacokinetic and pharmacodynamic data analysis in Microsoft Excel. Comput. Methods Programs Biomed. 99:306-314.

Zheng, S., Matzneller, P., Zeitlinger, M., Schmidt, S., 2014. Development of a Population Pharmacokinetic Model Characterizing the Tissue Distribution of Azithromycin in Healthy Subjects. Antimicrob. Agents Chemother. 58:66756684. 